\title{
Global Handwashing Day 2012: a qualitative content analysis of Chinese social media reaction to a health promotion event
}

\author{
Isaac Chun-Hai Fung, a Jingxian Cai, ${ }^{b}$ Yi Hao, ${ }^{b}$ Yuchen Ying, ${ }^{c}$ Benedict Shing Bun Chan, ${ }^{d}$ Zion Tsz Ho Tse and \\ King-Wa Fu \\ Correspondence to Isaac Chun-Hai Fung (email: cfung@georgiasouthern.edu)
}

Background: Global Handwashing Day (GHD) is a handwashing promotion campaign organized by the Global PublicPrivate Partnership of Handwashing with Soap. In China, it has been promoted by the Chinese public health authorities, international organizations and multinational corporations through various channels including social media such as Sina Weibo, the leading Chinese microblogging site similar to Twitter. The objective of this study is to qualitatively assess Chinese social media users' reactions to a health promotion campaign using Global Handwashing Day (GHD) 2012 as an example.

Methods: We conducted a qualitative content analysis of 552 Weibo posts generated on GHD 2012 by Weibo users with 1000 or more followers with the Chinese keyword for "handwashing". We categorized the Weibo posts into groups by keywords that frequently appeared in the data set. These groups were either exact reposts of an original post, or they conveyed similar information.

Results: We observed the interconnections between traditional media and social media in handwashing promotion. Social media were found to serve as amplifiers of contents provided by traditional media. We observed the contextualization of global hygiene messages in a unique national social media market in China.

Discussion: Our study showed that social media and traditional media are two interconnected arms of the GHD campaign in China. Our analysis demonstrated that public health campaigns in China can be evaluated using social media data. The themes and topics identified in this study will help public health practitioners evaluate future social media handwashing promotion campaigns.

$\mathrm{H}$ andwashing with soap has been shown to reduce the risk of diarrhoeal diseases and respiratory diseases. ${ }^{1-4}$ However, handwashing compliance at critical times (for example, before eating and after defecation) in many parts of the world is far from ideal. Gaps between knowledge and practice of handwashing persist. $^{5}$

The Global Public-Private Partnership of Handwashing with Soap (PPPHW) was founded in 2001 to promote handwashing with soap across the globe. ${ }^{6}$ It is a "coalition of international stakeholders", including the United Nations Children's Fund (UNICEF), government agencies, universities, nongovernmental organizations and representatives of the private sector. ${ }^{6}$ One of the key events that PPPHW organizes annually is Global Handwashing Day (GHD). The first GHD was organized on 15 October 2008 when more than 120 million children in 73 countries across five continents were mobilized to wash their hands with soap. ${ }^{6}$ Since then, 15 October has been designated as GHD, and handwashing promotion activities are organized globally on that day each year.

Social media have been used by community-based organizations $^{7}$ and government agencies ${ }^{8,9}$ to promote

\footnotetext{
Department of Epidemiology, Jiann-Ping Hsu College of Public Health, Georgia Southern University, Statesboro, Georgia, United States of America.

Department of Biostatistics, Jiann-Ping Hsu College of Public Health, Georgia Southern University, Statesboro, Georgia, United States of America.

Department of Computer Science, The University of Georgia, Athens, Georgia, United States of America.

Department of Religion and Philosophy, Hong Kong Baptist University; Department of General Education, School of Humanities and Social Science, Hang Seng Management College, Hong Kong Special Administrative Region, China.

College of Engineering, The University of Georgia, Athens, Georgia, United States of America.

Journalism and Media Studies Centre, The University of Hong Kong, Hong Kong Special Administrative Region, China

Submitted: 23 April 2015; Published: 9 July 2015

doi: 10.5365/wpsar.2015.6.2.003
} 
Figure 1. Schematic of our data collection, sampling criteria, syntax analysis and content analysis

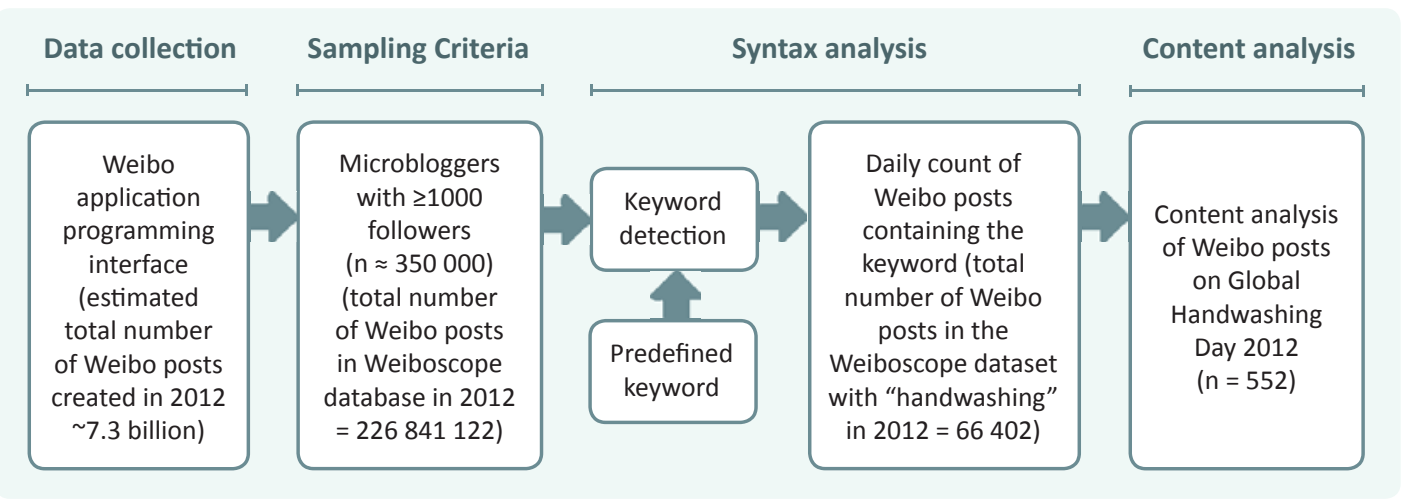

Note: The estimated total number of Weibo posts created in 2012 was based on a random sampling study by Fu \& Chau (2013). ${ }^{13}$

health. Examples of social media include Facebook, the world's leading social networking site, ${ }^{10}$ and Twitter, an online platform that allows users to post and repost 140-character-long messages online to the public. However, social media use in health promotion in many circumstances has remained uni-directional so that information flows only from public health professionals to the audience, ${ }^{7}$ as observed in a recent study of Twitter use in Breast Cancer Awareness Month. ${ }^{11}$ Raising health awareness and promoting healthy behaviours via social media remains a practical challenge for many organizations.

Weibo is the Chinese name for online platforms that provide microblogging services similar to Twitter. Weibo allows users to post publicly accessible messages of a maximum length of 140 Chinese characters via computers, tablets and smartphones. As Twitter is not accessible in mainland China, Weibo is popular as an alternative. Sina Weibo, the largest provider of Weibo services in China, claimed to have more than 500 million registered users as of December 2012. ${ }^{12}$ Meanwhile, $98 \%$ of Weibo users were from mainland China, according to a random sampling study. ${ }^{13}$ Weibo has been instrumental in online discussion of political and social events in China, ${ }^{14}$ responses to natural disasters ${ }^{15}$ and suicide prevention. ${ }^{16}$ Conversations about various aspects of health are common on Weibo. ${ }^{17}$ Weibo has also been a useful indicator of Chinese citizens' reaction to infectious disease outbreaks. ${ }^{18}$ Given its unique characteristics, Weibo facilitates study of the online reaction to a health promotion campaign in a well-defined population.

The purpose of this study is to provide a qualitative content analysis of Weibo posts that helps generate hypotheses about social media health promotion. It will serve as a starting point for future studies that can test these hypotheses.

\section{METHODS}

\section{Data acquisition and sampling}

Weibo data were collected through the Weiboscope project, as reported elsewhere. ${ }^{14,18,19}$ Through a systematic search of the Sina Weibo user database via the Sina Weibo Application Programming Interface (API), we generated a list of about 350000 indexed microbloggers who had 1000 or more followers when the project began data collection in 2011. We retrieved their Weibo posts regularly via API over the year of 2012. We selected a high-follower-count sample for two reasons. First, Weibo users with a high number of followers are more influential than those with a low follower count, and they frequently attract disproportionately large attention from the public. ${ }^{13}$ Second, spam Weibo accounts are very common in China, ${ }^{20}$ and our sampling methods minimize their influence (Figure 1). Our data set covered the year of 2012, and it is publicly available online. ${ }^{21}$ For the purpose of this study, we only performed qualitative analysis on the data from 15 October 2012 containing the Chinese keyword for "handwashing."

The raw Weibo data were acquired in CommaSeparated Values (CSV) format. ${ }^{14}$ The content of the Weibo posts as well as their metadata (e.g. their date of creation and the user IDs) were then available for secondary analysis. De-identification of the user IDs was performed through a process known as "hashing" (conversion into a different string of characters). The properties of each file were recorded in the first line, 
Figure 2. Daily count of Weibo posts that contained the Chinese keyword for handwashing in the Weiboscope database, 2012

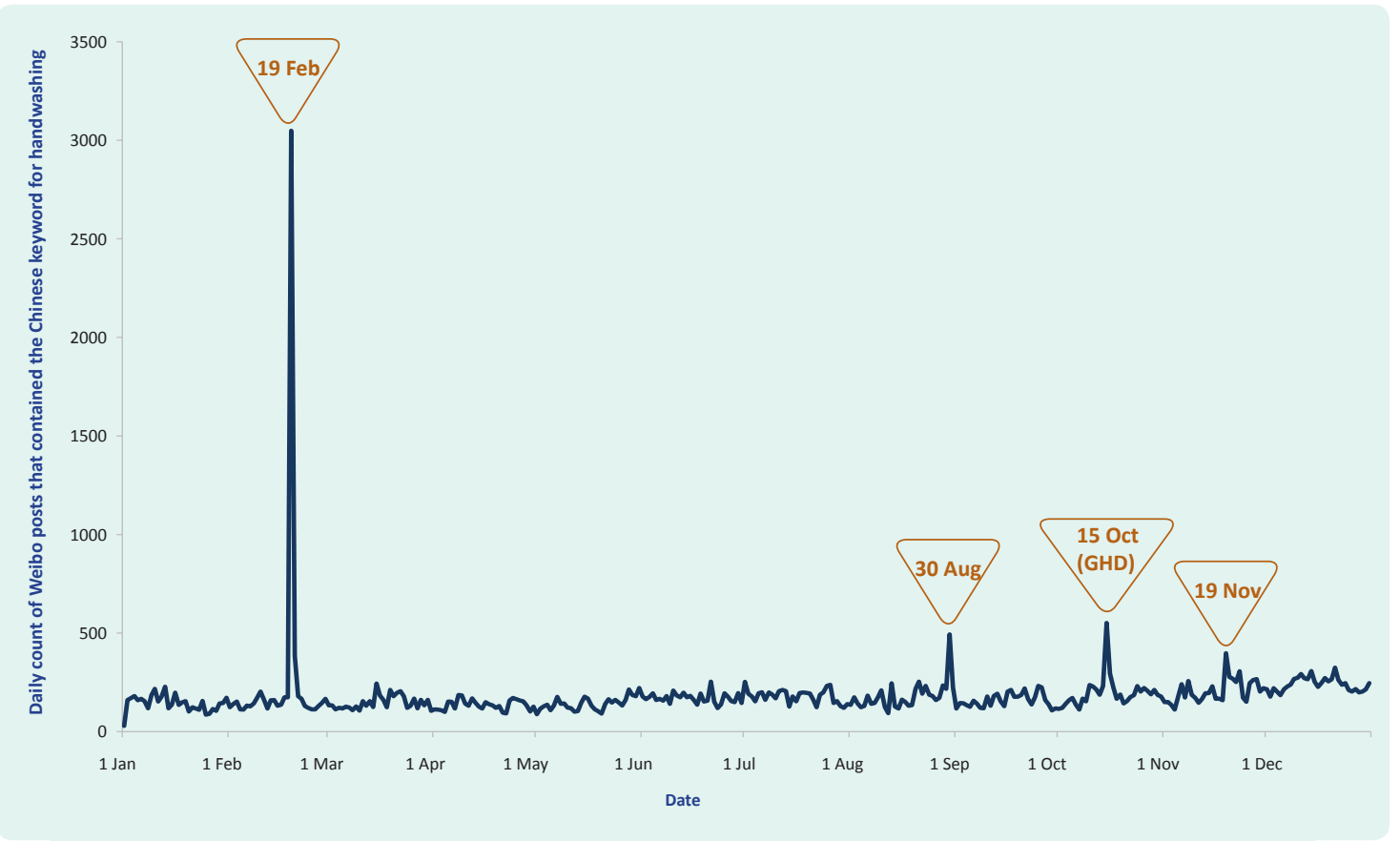

GHD, Global Handwashing Day.

followed by the Weibo post content. ${ }^{18}$ This protocol of data processing and anonymization was approved by the Human Research Ethics Committee for Non-Clinical Faculties, The University of Hong Kong (EA440512) and by the Institutional Review Board, Georgia Southern University (H14167).

\section{Keyword detection}

A string-searching algorithm was used in our keyword detection. We searched the Chinese keyword for handwashing in the data set through a Python-based platform described in a previous study. ${ }^{18}$ "The stringsearching algorithm in Python was implemented in C programming language. Python's multiprocessing library was used to process multiple files in parallel to accelerate the search." ${ }^{18}$ Every Weibo post in the data set was searched, and we recorded if the keyword occurred in the data file (Figure 1). The daily count of Weibo posts mentioning handwashing is presented in Figure 2. We note that the Chinese keyword for handwashing may be combined with other Chinese characters to form other terms such as toilet/restroom or hand soap bottle which will reduce the positive predictive value (or precision) of our keyword. (For the original Chinese characters please refer to Table S1, Online Supplementary Materials.)

Our data set included original Weibo posts and reposts, i.e. forwarded messages posted by someone else, or retweets in Twitter parlance. (Please note one important difference between Weibo re-posts and Twitter re-tweets. A re-tweet on Twitter must be limited to 140 letters, including the content of the original tweet. In contrast, Sina Weibo allows users to re-post a Weibo post and add another 140 Chinese characters.)

\section{Qualitative content analysis}

We performed a qualitative content analysis of the Weibo posts mentioning handwashing. First, preliminary analysis was performed by identifying certain keywords that frequently appeared in the data set and noting their frequency of appearance. Next, the Weibo posts were categorized into groups. These groups were either exact reposts of an original post or they conveyed similar information. Topics of interest that might help generate hypotheses for future studies were then identified. We also visited the Weibo home pages of the key stakeholders of GHD 2012 in China, including the Chinese public 
Table 1. Examples of Weibo posts explaining the origin and purpose of GHD.*

\begin{tabular}{|c|c|c|}
\hline & English translation & Notes \\
\hline Example 1 & $\begin{array}{l}\text { [Global Handwashing Day] Global Handwashing Day was initiated in } 2008 \\
\text { by the Global Public-Private Partnership for Handwashing with Soap to call } \\
\text { upon the nations of the world to hold activities promoting handwashing with } \\
\text { soap on } 15 \text { October every year. According to the World Health Organization, } \\
\text { "correct handwashing" needs to satisfy four criteria: handwashing before } \\
\text { meals, after defecation and touching coins, etc.; using running water; using } \\
\text { soap or other cleansing products; handwashing for no less than } 20 \text { seconds. } \\
\text { Do you wash your hands often? }\end{array}$ & $\begin{array}{l}\text { Altogether there were } 13 \text { Weibo } \\
\text { posts in our data set that } \\
\text { mentioned PPPHW. }\end{array}$ \\
\hline Example 2 & $\begin{array}{l}\text { [Global Handwashing Day] } 15 \text { October is Global Handwashing Day. Even } \\
\text { though handwashing is a small thing in daily life, this simple and important } \\
\text { action has a very significant effect on raising people's hygiene awareness and } \\
\text { preventing diseases. Kids, especially, must develop good handwashing habits } \\
\text { and love handwashing. Dear everyone, is your handwashing method correct? } \\
\text { If it isn't you need to learn it well. }\end{array}$ & $\begin{array}{l}\text { There were another seven } \\
\text { Weibo posts in our data set that } \\
\text { were very similar to this post. }\end{array}$ \\
\hline
\end{tabular}

* The original Chinese posts and the emotion icons therein can be found in Alternative Table 1 in the Online Supplementary Materials.

GHD, Global Handwashing Day; PPPHW, Private-Public Partnership of Handwashing with Soap.

health agencies, UNICEF and three multinational or national corporations that sold hand hygiene products in China. In this paper, the identities of only these large entities are revealed and discussed. No individual Weibo users are identified.

\section{RESULTS}

\section{Description of peaks of handwashing Weibo posts}

In 2012, there were four major peaks in our Weibo data set with the keyword for handwashing (Figure 2). The peak on 19 February 2012 was generated by reposts of news about the extravagant cost of accessories on certain Chinese high-speed trains, including an expensive hand soap bottle. The peak on 30 August 2012 was generated by news about an $E$. coli concentration in ice-cream of a certain brand exceeding established standards. Because the news proposed that "incomplete handwashing" by production personnel was a potential source of secondary contamination, a surge in Weibo posts mentioning "handwashing" was observed. ${ }^{22}$ The peak on World Toilet Day (19 November 2012) was unrelated to that campaign but related to a news report on that day about the suicide of a university student who dropped to her death out of a restroom window in a university building. ${ }^{23}$

\section{Content analysis}

In total, 552 Weibo posts on GHD by keyword search were identified. Of these 552 posts, 82 (15\%) were deemed irrelevant as the keyword for handwashing was combined with other Chinese characters to form other terms, such as washing basins, toilet/restroom and hand soap.

\section{Basic information about GHD}

There were 13 Weibo posts $(13 / 552,2.4 \%)$ that mentioned PPPHW by either its Chinese name or its English acronym (Table 1). These posts provided correct information about the origin of GHD. There were another seven Weibo posts in our data set that suggested that the GHD was established by the World Health Organization on 13 October 2005 (Table S2 in Online Supplementary Materials).

We also found that while 212 posts $(212 / 552$, $38.4 \%$ ) carried the official Chinese name of Global Handwashing Day, 17 (3.1\%) posts mentioned "World Handwashing Day" and 32 (5.8\%) posts mentioned "International Handwashing Day".

\section{GHD promotional events, videos and commercial advertisements}

We identified 56 Weibo posts $(56 / 552,10.1 \%)$ that were related to the official GHD promotional event organized by the Chinese Government. The guests of honour were an Olympic gold medallist and his mother. UNICEF also used Weibo to promote their GHD videos on the Chinese equivalent of YouTube (Youku) that featured a movie star. Table 2 illustrates the reposting sequence of the original post by UNICEF with some examples. Commercial firms 
Table 2. An example of a chain of reposts of UNICEF Weibo post on GHD 2012*

\begin{tabular}{|c|c|c|}
\hline & English translation of sample Weibo posts & Notes \\
\hline Post & $\begin{array}{l}\text { Today is \#Global Handwashing Day\#. According to the estimates of the World } \\
\text { Health Organization, every year in China, about } 40000 \text { children under the } \\
\text { age of five die of diarrhoea. Handwashing with soap and water at critical } \\
\text { moments, such as before meals or after defecation, would help reduce the } \\
\text { incidence of diarrhoeal diseases by over } 40 \% \text { and the incidence of respiratory } \\
\text { diseases by nearly } 25 \% \text {. Let us follow Chen Kun to do a handwashing } \\
\text { exercise! Learn how to wash your hands in } 50 \text { seconds. ( http://t.cn/h4XSzE) }\end{array}$ & $\begin{array}{l}\text { Original post by UNICEF (http://www. } \\
\text { weibo.com/1749268803/zOyXM6jtH, } \\
\text { accessed } 15 \text { June 2015). }\end{array}$ \\
\hline Repost 1 & $\begin{array}{l}\text { [Global Handwashing Day] Today is Global Handwashing Day. Quickly, } \\
\text { together with UNICEF China Ambassador Chen Kun: correct handwashing, } \\
\text { building health by hands! (http://t.cn/h4XSzE) }\end{array}$ & $\begin{array}{l}\text { Repost by United Nations (http://weibo. } \\
\text { com/1709157165/zOAvf6wn1, accessed } \\
15 \text { June 2015). }\end{array}$ \\
\hline Repost 2 & $\begin{array}{l}\text { Children, have you washed your hands? If not, hurry up and wash before you } \\
\text { sleep. }\end{array}$ & $\begin{array}{l}\text { A Weibo user's repost of United Nations' } \\
\text { repost of UNICEF's post. }\end{array}$ \\
\hline
\end{tabular}

* The original Chinese posts and the emotion icons can be found in Alternative Table 2 in the Online Supplementary Materials.

GHD, Global Handwashing Day.

also promoted GHD 2012 via Weibo. (Supplementary section 1, Tables S3 and S4 in Online Supplementary Materials.)

\section{A report on a survey of handwashing behaviour}

We identified 50 Weibo posts $(50 / 552,9.1 \%)$ in our data set that quoted a report from the official national paper of the Chinese government (People's Daily) on a survey that found that only $7 \%$ of the respondents washed hands in all seven circumstances specified in the survey (after defecation, before meals, after changing nappies, after meals, after touching pets, after touching money and after sneezing). We identified another 19 Weibo posts $(19 / 552,3.4 \%)$ that mentioned two other handwashing-related surveys (Supplementary section 2 and Table S6 in Online Supplementary Materials).

\section{Washing hands correctly}

\section{Four criteria of correct handwashing}

Several Weibo posts mentioned a so-called "World Health Organization's (WHO) four criteria on correct handwashing" (the WHO guidelines have six steps):

(1) handwashing before meals, after defecation and touching coins, etc.; (2) using running water; (3) using soap or other cleansing products; and (4) handwashing for no less than 20 seconds. For example, we identified 52 posts $(52 / 552,9.4 \%)$ that mentioned "20 seconds". Forty-one posts mentioned all four criteria, five mentioned three criteria and six posts mentioned just one criterion (handwashing for no less than 20 seconds) (Table 1, Example 1).

\section{Six steps of handwashing}

The "Six Steps of Handwashing," first originated in an experiment by Ayliffe et $\mathrm{al}^{24}$ are now being promoted globally as a standard handwashing method. We identified 29 Weibo posts $(29 / 552,5.2 \%)$ that mentioned six steps of handwashing (Examples 1 and 2 in Table 3). Interestingly, this method, though it originated in the United Kingdom, gained a new nickname in China. We identified posts that described the six steps of handwashing as the "Buddha's Palm", literally "Tathāgata Palm" (10 posts) or "Tathāgata Divine Palm" (4 posts) (Examples 2 and 3 in Table 3 ). This was originally a fictional martial arts move that appeared in movies. The People's Daily released a poster on their official Weibo with the title "Practice 'Buddha's Palm' diligently. Wash hands and keep healthy" and described the six steps of handwashing in a poem ${ }^{25}$ (Table S6 in Online Supplementary Materials). This is an example of how a hygiene practice adopted by the global medical community can be adapted and contextualized in a health promotion campaign in a particular culture.

\section{Importance and benefits of handwashing}

Weibo posts described the importance and benefits of handwashing. Examples include the "very important effect on raising people's hygiene awareness and preventing diseases" (Table 1, Example 2), the reduction of "the incidence of diarrhoeal diseases by over $40 \%$ and the incidence of respiratory diseases by nearly 25\%" (Table 2), and that "correct handwashing is more costeffective than vaccines or any other health interventions" (Table S7, Example 1 in Online Supplementary Materials). 
Table 3. Examples of Weibo posts that mentioned or alluded to the six steps of handwashing*

\begin{tabular}{|c|c|c|}
\hline & English translation of sample Weibo posts & Notes \\
\hline Example 1 & \multicolumn{2}{|l|}{$\begin{array}{l}\text { [Today is "Global Handwashing Day"] Have you washed your hands? } \\
\text { Experts point out that regular handwashing removes only } 18 \% \text { of bacteria. } \\
\text { The correct method: (1) rub palm to palm with fingers closed together; } \\
\text { (2) rub palm to back of the hand with fingers interlaced, and then repeat } \\
\text { after changing hand positions; (3) rub palm to palm with fingers interlaced; } \\
\text { (4) bend fingers, rub the finger joints in the palm of the other hand; } \\
\text { (5) clasp the thumb of the left hand with the right hand and rub in a } \\
\text { rotational movement; (6) rub the finger tips in a closed position in the } \\
\text { opposite palm in a circular motion. }\end{array}$} \\
\hline Example 2 & $\begin{array}{l}\text { \#World Handwashing Day\# [Today have you washed your hands?] Today } \\
\text { is Global Handwashing Day. Hands are the carriers of bacteria and virus. } \\
\text { Through direct contacts or through indirect contact in public venues, } \\
\text { hands can spread germs from one person to another. How do you wash } \\
\text { hands correctly? Quickly, come and take a look at the "Buddha's Palm" } \\
\text { style six steps of handwashing. Let us wash hands correctly. Touch the } \\
\text { world without worries. P.S. Lunchtime is approaching. Remember to wash } \\
\text { your hands before every meal! }\end{array}$ & $\begin{array}{l}\text { The link is a United Nations } \\
\text { Children's Fund video featuring } \\
\text { Chen Kun (a famous Chinese } \\
\text { actor) and children washing } \\
\text { hands using the correct methods } \\
\text { (http://v.youku.com/v_show/id_- } \\
\text { XMjEOMTI2NTAw.html, accessed } \\
15 \text { June 2015). }\end{array}$ \\
\hline Example 3 & $\begin{array}{l}\text { Youth, have you washed your paws? Practice "Buddha’s Palm" diligently. } \\
\text { Wash hands and keep healthy! }\end{array}$ & $\begin{array}{l}\text { "Buddha's Palm" became a } \\
\text { synonym of the six steps of } \\
\text { handwashing. }\end{array}$ \\
\hline \multicolumn{3}{|c|}{ The original Chinese posts and the emotion icons therein can be found in Alternative Table 3 in the Online Supplementary Materials. } \\
\hline \multicolumn{3}{|c|}{$\begin{array}{l}\ddagger \text { Literally "Tathāgata Palm". "Buddha's Palm" is a fictional martial arts move that appears in popular kungfu (martial arts) movies and wuxia (martial } \\
\text { hero) novels. }\end{array}$} \\
\hline
\end{tabular}

\section{Individuals' comments}

Apart from public health agencies, commercial firms and mass media outlets, some Weibo users also generated their own comments in reaction to GHD. To illustrate the diversity of contents, we chose five posts as examples (see Table $\mathrm{S} 8$ in Online Supplementary Materials): (1) an example of someone's personal experience after attending four sessions of hand hygiene training; (2) a creative Weibo post promoting GHD; (3) a sarcastic comment on GHD; (4) a Weibo post that freely associated GHD with a scene in a popular novel; and (5) a Weibo post that took the opportunity to persuade fugitives and potential criminals to stop committing crimes. These examples highlight how a successful public health campaign may draw attention from a variety of people, who may or may not interpret it in the way the organizers of the campaign initially envisioned.

\section{DISCUSSION}

We performed a qualitative content analysis of 552 Weibo posts generated on GHD 2012 by Weibo users who had 1000 or more followers. We identified various GHD-related themes and topics, including basic information about GHD; promotional events, multimedia and commercial advertisements; and health information such as prevalence of handwashing behaviour, the correct handwashing method and the importance and benefits of handwashing. We also identified some comments created by individual Weibo users.

As a global health promotion initiative, GHD 2012 was chosen as a case study because this health promotion campaign was promoted by the Chinese Government and its partners through a few clearly identified activities and multimedia materials within a well-defined time frame. ${ }^{26}$ It was advertised via social media as well as other traditional mass media. Moreover, PPPHW had developed social media guidelines for its partners to use in GHD promotion.

We found that Weibo was used as a means to disseminate information by various stakeholders of GHD in China, including the Chinese public health authorities, UNICEF and a few corporations that produced hand hygiene products. Weibo posts formed part of the overall health communication strategy to raise GHD awareness together with other promotional events, TV shows, guest celebrities, videos and songs. Posting events in real time in Weibo, and posts having links to instructional videos or songs about handwashing helped the promotion.

Social media can serve as amplifiers of contents provided in traditional mass media such as radio, television and print. $^{11,27}$ As illustrated by the Weibo 
posts about the survey on the prevalence of handwashing behaviour among Chinese urban residents, newspapers used Weibo to disseminate headlines and provide links to their newspaper articles (Table S6 in Online Supplementary Materials). Traditional media may report on contents that are originated on social media. ${ }^{28}$ While our analysis suggested that social media were used by organizations as a means for health promotion, it did not provide evidence that social media had replaced traditional media in health communications.

Social media facilitate evaluation of health communications campaigns. In the past, health communications via mass media could be evaluated through surveys such as TV ratings. The dissemination process of Twitter or Weibo messages can also be documented and analysed now. The availability of social media data allows public health professionals to evaluate their health communication campaigns in a timely manner through both quantitative and qualitative analyses. By identifying the themes and topics of social media contents, health communication professionals may be able to focus their efforts on creating and disseminating contents that attract more attention. In the future, researchers can also investigate how photo and video links in Weibo posts may attract more attention and determine the characteristics of Weibo users who are more likely to repost health communication messages.

Social media in mainland China form a distinctive national market as there is only limited access to certain global social media (e.g. Facebook, Twitter and Youtube, etc.). China-based, Chinese-language social media such as Sina Weibo and Youku have attained phenomenal success in this unique environment. ${ }^{29}$ However, their role in health communications in China has not yet attracted much scholarly attention. Future research that compares Weibo with Twitter, or Youku with Youtube, will further reveal the similarities and differences between these platforms and how health information disseminates and evolves in a distinctive national Internet user community. ${ }^{28}$

Social media are lenses through which contextualization of global public health messages can be observed and documented. GHD as a global initiative relies on national and local partners to promote handwashing. Handwashing promotional messages that originated from international organizations were contextualized by the Chinese stakeholders into culturally adapted messages. They were then disseminated from the capital to the rest of China via China-based social media. The re-invention of the six steps of handwashing as the "Buddha's Palm", as found in the Weibo posts, is a good illustration. Further analyses on the contextualization of these promotional messages will inform public health professionals on how they can better contextualize health communication in today's digital age. ${ }^{31}$

As the data were originally collected for a different study, ${ }^{14}$ we did not define our operational sampling parameters to optimize collection of data pertaining to any specific health-related keywords. Our data set only comprised 350000 Weibo users who had 1000 or more followers. Among these users, around 5000 were Chinese dissident writers, journalists and scholars; another 38000 were users with an authenticated status having more than 10000 followers. ${ }^{14,19}$ The study sample constituted less than $1 \%$ of all registered users of Sina Weibo. ${ }^{13}$ Nonetheless, the study samples represent the most influential Weibo users who contributed the most content in Weibo. According to a random sampling study, $5 \%$ of Weibo accounts generate $80 \%$ of the original posts, and over $50 \%$ of Weibo accounts never post anything. ${ }^{13}$

The Chinese keyword for handwashing may be combined with other Chinese characters to form other terms as demonstrated in two of the four peaks discussed in the Results section. Eighty-two (15\%) of the 552 posts belonged to this category in which the Chinese characters for handwashing were part of a longer term; those posts were irrelevant to GHD. The positive predictive value was found to be about $85 \%$ for handwashing in this study.

A certain level of subjectivity cannot be avoided in our qualitative analysis. Nonetheless, our goal is to identify topics and themes that will facilitate future research in digital health communications and, in particular, handwashing promotion. Categories created in this paper can be adapted to code Weibo posts and Twitter tweets in future studies.

While handwashing Weibo posts might lead to increased awareness and practice of handwashing among its users, our analysis did not provide any direct evidence to support that. Obtaining such evidence requires surveys of knowledge, attitudes and perceptions of hand hygiene, ${ }^{32,33}$ structured observation studies ${ }^{34,35}$ 
and/or video surveillance ${ }^{36}$ of handwashing behaviours in community settings. Furthermore, as with other social media, Weibo users are, in general, younger and more urban. ${ }^{37}$ Reaching the rural poor, especially the elderly in China, as in any middle or low-income country, will require other creative means.

We performed a qualitative analysis of the content of Weibo posts about GHD 2012 to identify topics and themes of handwashing promotion in China. Our study showed that social media and traditional media are two interconnected arms of the GHD campaign in China. We documented the contextualization of global handwashing messages and their dissemination to audiences in a national social media market that is protected from international competition. Our analysis demonstrated that public health campaigns in China can be evaluated using social media data. ${ }^{11}$ Our analysis serves as one step towards future comparative social media studies of global health promotion campaigns.

\section{Conflicts of interest}

None declared.

\section{Funding}

None.

\section{Acknowledgement}

The authors thank Mr Chung-Hong Chan for questions regarding emotion icons on Weibo. The authors thank Dr Pavani Ram and Ms Hanna Woodburn for helpful discussion about handwashing. Jingxian Cai and Yi Hao thank Jiann-Ping Hsu College of Public Health for their graduate assistantships.

Jingxian Cai and Yi Hao contributed equally to this paper. Zion Tsz Ho Tse and King-Wa Fu are co-senior authors of this paper.

\section{References:}

1. Curtis V, Cairncross S. Effect of washing hands with soap on diarrhoea risk in the community: a systematic review. The Lancet Infectious Diseases, 2003, 3:275-281. doi:10.1016/S14733099(03)00606-6 pmid:12726975

2. Ryan MA, Christian RS, Wohlrabe J. Handwashing and respiratory illness among young adults in military training. American Journal of Preventive Medicine, 2001, 21:79-83. doi:10.1016/S07493797(01)00323-3 pmid:11457626
3. Cairncross S. Handwashing with soap - a new way to prevent ARIs? Tropical Medicine \& International Health, 2003, 8:677-679. doi:10.1046/j.1365-3156.2003.01096.x pmid: 12869087

4. Luby SP et al. Effect of handwashing on child health: a randomised controlled trial. Lancet, 2005, 366:225-233. doi:10.1016/ S0140-6736(05)66912-7 pmid:16023513

5. Rabbi SE, Dey NC. Exploring the gap between hand washing knowledge and practices in Bangladesh: a cross-sectional comparative study. BMC Public Health, 2013, 13:89. doi:10.1186/1471-2458-13-89 pmid:23363772

6. The Global Public-Private Partnership for Handwashing (PPPHW). (http://globalhandwashing.org/, accessed 25 June 2015).

7. Ramanadhan $S$ et al. Social media use by community-based organizations conducting health promotion: a content analysis. BMC Public Health, 2013, 13:1129. doi:10.1186/1471-245813-1129 pmid:24313999

8. Harris JK et al. Local health department use of twitter to disseminate diabetes information, United States. Preventing Chronic Disease, 2013, 10:E70. doi:10.5888/pcd10.120215 pmid:23639765

9. Harris JK, Snider D, Mueller N. Social media adoption in health departments nationwide: the state of the states. Frontiers in Public Health Services and Systems Research, 2013, 2:5.

10. Mander J. GWI Social: GlobalWeblndex's quarterly report on the latest trends in social networking (Q4 2014). London, GlobalWeblndex, 2015.

11. Thackeray $\mathrm{R}$ et al. Using Twitter for breast cancer prevention: an analysis of breast cancer awareness month. BMC Cancer, 2013, 13:508. doi:10.1186/1471-2407-13-508 pmid:24168075

12. Mozur P. How many people really use Sina Weibo? Beijing, Wall Street Journal China, 2013 (http://blogs.wsj.com/ chinarealtime/2013/03/12/how-many-people-really-use-sinaweibo/, accessed June 28, 2015).

13. Fu KW, Chau M. Reality check for the Chinese microblog space: a random sampling approach. PLOS ONE, 2013, 8:e58356. doi:10.1371/journal.pone.0058356 pmid:23520502

14. Fu KW, Chan $\mathrm{CH}$, Chau M. Assessing censorship on microblogs in China: discriminatory keyword analysis and the real-name registration policy. Internet Computing, IEEE, 2013, 17:42-50. doi:10.1109/MIC.2013.28

15. Fu KW et al. Newspaper coverage of emergency response and government responsibility in domestic natural disasters: China-US and within-China comparisons. Health Risk \& Society, 2012, 14:71-85. doi:10.1080/13698575.2011.641521

16. Fu KW et al. Responses to a self-presented suicide attempt in social media: a social network analysis. Crisis. Journal of Crisis Intervention and Suicide Prevention, 2013, 34:406-412. doi:10.1027/0227-5910/a000221

17. Wang S, Paul MJ, Dredze M. Exploring health topics in Chinese social media: an analysis of Sina Weibo. AAAl Workshop: the world wide web and public health intelligence, 2014 (http:// www.cs.jhu.edu/ mdredze/publications/2014_w3phi_weibo.pdf, accessed 15 June 2015).

18. Fung IC-H et al. Chinese social media reaction to the MERS-CoV and avian influenza $\mathrm{A}(\mathrm{H} 7 \mathrm{~N} 9)$ outbreaks. Infectious Diseases of Poverty, 2013, 2:31. doi:10.1186/2049-9957-2-31 pmid:24359669

19. Fung $\mathrm{IC}-\mathrm{H}$ et al. Chinese social media reaction to information about 42 notifiable infectious diseases. PLOS ONE, 2015, 10:e0126092. doi:10.1371/journal.pone.0126092 pmid:25946020 
20. Yu LL, Asur S, Huberman BA. Artificial inflation: the real story of trends and trend-setters in Sina Weibo. Amsterdam, Privacy, Security, Risk and Trust (PASSAT), 2012 International Conference on and 2012 International Conference on Social Computing (SocialCom), 2012, 514-519 (http://ieeexplore.ieee.org/xpl/ articleDetails.jsp?arnumber $=6406395$ \&punumber\%3D6403618 \%26filter\%3DAND\%28p_IS_Number\%3A6406254\%29\%26pag eNumber\%3D3, accessed $2 \overline{5}$ June 2015).

21. Open WDA. Hong Kong, Journalsim and Media Studies Centre, The University of Hong Kong, 2013 (http://weiboscope.jmsc.hku. hk/datazip/, accessed 25 June 2015).

22. Liang S. DQ ice-cream E. coli concentration 10 times the standards; Cold stone, Donut King are also blacklisted [In Chinese]. Shanghai Eastern Daily, 30 August 2012 (http://sh.eastday.com/m/20120830/u 1 a6823336.html, accessed 30 June 2015]

23. A female student went to dine with her teachers, fell and died. The university said the journalists created trouble for themselves [In Chinese]. Sina News, 18 November 2012 (http://news. sina.com.cn/s/2012-11-18/232625606163.shtml, accessed 30 June 2015).

24. Ayliffe GA, Babb JR, Quoraishi AH. A test for 'hygienic' hand disinfection. Journal of Clinical Pathology, 1978, 31:923-928. doi:10.1136/jcp.31.10.923 pmid:10155

25. People's Daily (official Weibo account). Practice "Buddha's Palm" diligently: wash hands and keep healthy. Beijing, 15 October 2012 (http://www.weibo.com/2803301701/zOzo zizjy\#_rnd1405090301269, accessed 30 June 2015).

26. About the initiation of the promotional activities of "Global Handwashing Day" 2012, a notice by the Office of the National Committee for Patriotic Health Campaign [A repost by BaoTou Center for Disease Control and Prevention]. Beijing, Office of the National Committee for Patriotic Health Campaign, 2012 (http://www.btscdc.org.cn/article/1237.html, accessed 30 May 2015).

27. Fu K-W, Chau M. Use of microblogs in grassroots movements in China: exploring the role of online networking in agenda-setting. Journal of Information Technology \& Politics, 2014, 11:309328. doi:10.1080/19331681.2014.909344

28. McCoy T. How 'Ice Bucket' became a fundraising juggernaut. Washington, DC, Washington Post, 18 August 2014 (http://www. washingtonpost.com/news/morning-mix/wp/2014/08/18/theice-bucket-challenges-remarkable-evolution-into-a-fundraisingjuggernaut/, accessed 25 June 2015).

29. White J, Fu K-W, Benson B. Social media: an ill-defined phenomenon. Online Communities and Social Computing, 2013, 8029:422-431.

30. Gao $Q$ et al. A comparative study of users' microblogging behavior on Sina Weibo and Twitter. In: Masthoff J et al (eds). User modeling, adaptation, and personalization. New York, Springer, 2012, 88-101.

31. Boyd D. Social network sites as networked publics: affordances, dynamics, and implications. In: Papacharissi Z (ed). Networked self: identity, community, and culture on social network sites. Routledge, 2010, 39-58.

32. Tao SY et al. Handwashing behaviour among Chinese adults: a cross-sectional study in five provinces. Public Health, 2013, 127:620-628. doi:10.1016/j.puhe.2013.03.005 pmid:23790806

33. Sibiya JE, Gumbo JR. Knowledge, attitude and practices (KAP) survey on water, sanitation and hygiene in selected schools in Vhembe District, Limpopo, South Africa. International Journal of Environmental Research and Public Health, 2013, 10:22822295. doi:10.3390/ijerph10062282 pmid:23736657

34. Ram PK et al. Is structured observation a valid technique to measure handwashing behavior? Use of acceleration sensors embedded in soap to assess reactivity to structured observation. The American Journal of Tropical Medicine and Hygiene, 2010, 83:1070-1076. doi:10.4269/ajtmh.2010.09-0763 pmid:21036840

35. Halder AK et al. Impact of duration of structured observations on measurement of handwashing behavior at critical times. BMC Public Health, 2013, 13:705. doi:10.1186/1471-2458-13-705 pmid:23915098

36. Pickering AJ et al. Video surveillance captures student hand hygiene behavior, reactivity to observation, and peer influence in Kenyan primary schools. PLOS ONE, 2014, 9:e92571. doi:10.1371/journal.pone.0092571 pmid:24676389

37. Statistical Report on Internet Development in China 2014 [in Chinese]. Beijing, China Internet Network Information Center, 2014 (http://www.cnnic.cn/, accessed 25 June 2015). 